\title{
Profile of Trastuzumab Deruxtecan in the
} Management of Patients with HER2-Positive

\section{Unresectable or Metastatic Breast Cancer: An Evidence-Based Review}

This article was published in the following Dove Press journal:

Breast Cancer: Targets and Therapy

\author{
Anna S Linehan $\mathbb{D}^{\prime}$ \\ Orla M Fitzpatrick' \\ Patrick G Morris ${ }^{1,2}$ \\ 'Cancer Clinical Trials and Research Unit, \\ Beaumont Hospital, Dublin, Ireland; \\ ${ }^{2}$ Department of Medical Oncology, Royal \\ College of Surgeons in Ireland, Dublin, \\ Ireland
}

\begin{abstract}
Trastuzumab deruxtecan is a novel antibody-drug conjugate for the treatment of advanced solid tumors, including breast cancer, which overexpress or have amplification of the human epidermal growth factor receptor 2 (HER2). The novel structure of this exciting new agent means that it can deliver a highly potent cytotoxic agent to HER2-expressing tissues resulting in selective killing of cancer cells. In phase I and II trials, trastuzumab deruxtecan has shown impressive response rates in heavily pretreated populations, including patients who had received prior treatment with trastuzumab emtansine, another highly active antibody-drug conjugate. The most common side effects are gastrointestinal and hematological. Importantly, a high rate of interstitial lung disease was seen in early trials, but this appears manageable in most patients with corticosteroids. In light of its efficacy, this promising new drug may change the treatment paradigm of HER2-positive breast cancer.

Keywords: antibody-drug conjugate, DS8201, HER2, trastuzumab deruxtecan, trastuzumab emtansine
\end{abstract}

\section{Background}

About $15-20 \%$ of all breast cancers overexpress or have gene amplification of the human epidermal growth factor receptor 2 (HER2). Historically, patients with these so-called HER2-positive cancers have had poor outcomes. However, the introduction of trastuzumab, the first targeted therapy for HER2 revolutionized the treatment of HER2-positive breast cancer. ${ }^{1}$ Subsequently, the advent of drugs such as pertuzumab and trastuzumab emtansine (T-DM1) has resulted in a substantial shift in the treatment paradigm from conventional chemotherapy to HER2-directed therapies and a median overall survival of $>50$ months. ${ }^{2,3}$

For patients with newly diagnosed metastatic HER2-positive breast cancer, the current standard of care is dual HER2 blockade with trastuzumab and pertuzumab in combination with taxane chemotherapy based on results from the CLEOPATRA study. ${ }^{4}$ This randomized phase III trial showed that the addition of pertuzumab to trastuzumab and docetaxel led to an improvement in the median overall survival from 40.8 months to 56.5 months. ${ }^{5}$ In the second line, treatment with the antibodydrug conjugate (ADC) trastuzumab emtansine is now standard practice. The evidence for this comes from the EMILIA trial which compared trastuzumab
Correspondence: Patrick G Morris Beaumont Hospital, Dublin, 9, Ireland Tel +353 I 8092010

Email patrickmorris@beaumont.ie
Breast Cancer: Targets and Therapy 2021:13 I5I-159 
emtansine with lapatinib and capecitabine in patients who had one prior line of treatment (which included trastuzumab) and found a median overall survival benefit of 5.8 months in favor of the trastuzumab emtansine arm. ${ }^{6}$

Beyond the second line, there is no universally agreed standard of care, and the most accepted strategy is to maintain HER2 blockade in combination with a chemotherapeutic agent. ${ }^{3}$ There has been extensive work on small molecule tyrosine kinase inhibitors of HER2 such as lapatinib and neratinib with their ability to cross the blood-brain barrier desirable in a population highly susceptible to CNS metastases. ${ }^{7-9}$ Although these agents are active, broad use is limited by toxicities such as diarrhea, which can be debilitating. More recently, tucatinib a newer highly selective tyrosine kinase inhibitor of HER2 was granted Food and Drug Association (FDA) approval in 2020 based on results from the phase III HER2CLIMB trial. In this study, patients who received tucatinib, trastuzumab, and capecitabine had an objective response rate of $40.6 \%$ versus $20.8 \%$ in those who received placebo, trastuzumab, and capecitabine. The median overall survival was 21.9 compared to 17.4 months for patients treated with placebo. ${ }^{10}$

Another novel agent in this setting is the Fc engineered anti-HER2 monoclonal antibody margetuximab. Margetuximab binds to the same subdomain as trastuzumab but with higher affinity to the activation Fc receptor $\mathrm{CD} 16 \mathrm{~A}$ and lower affinity to inhibitory $\mathrm{Fc}$ receptor CD32B leading to greater antibody cell-mediated cytotoxicity. The phase III SOPHIA trial of margetuximab plus chemotherapy versus trastuzumab plus chemotherapy yielded disappointing results with only a one-month progression-free survival benefit. ${ }^{11}$ Patients with CD16A$158 \mathrm{~F}$ allele may derive more benefit. There are several other HER2-targeted agents under investigation in early phase trials currently including the irreversible pan-ErbB tyrosine kinase inhibitor, pyrotinib and the antibody-drug conjugate trastuzumab duocarmazine. This article focuses on the development to date of the novel antibody-drug conjugate trastuzumab deruxtecan, one of the most exciting agents under development.

\section{Pharmacokinetics}

Trastuzumab deruxtecan (DS-8201) is an antibody-drug conjugate, which is composed of a three parts: a monoclonal antibody targeting HER2, a tetrapeptidebased linker which is cleavable, and a cytotoxic payload. $^{12}$ The antibody component seeks out the antigen- expressing tumor cells and the conjugate is then transported into the tumor cell by endocytosis. The linker, which is stable in plasma, is cleaved by proteases known as lysosomal cathepsins once in the cell, causing release of the cytotoxic drug. ${ }^{13}$ These cathepsins are up-regulated in cancer cells. ${ }^{14}$ This allows selective killing of the cancer cells. The cytotoxic payload is deruxtecan (DXd), a topoisomerase I inhibitor, which works by forming a stable complex with human DNA topoisomerase DNA and $I$ and induces DNA damage. ${ }^{13}$ It is potent, with the anti-proliferative effect being 6-fold the active metabolite of irinotecan (SN-38, which is used as the payload in sacituzumab govitecan). It has a short systemic elimination half-life of 5.7 days, which potentially reduces side effects. $^{15}$ It is not metabolized through the UGT1A1 gene; therefore, it is theoretically safer than other topoisomerase inhibitors which can lead to severe side effects such as diarrhea in those with a malfunction of the UGT1A1 gene. ${ }^{13}$

Another important aspect of trastuzumab deruxtecan is its high drug-to-antibody ratio (DAR), which at 8 , is significantly higher than other antibody-drug conjugates (for example, the DAR of trastuzumab emtansine is 3.5). There has been a suggestion that higher DARs result in higher plasma clearance and increased side effects, but the stability of the linker in this case appears to allow high efficacy without significant adverse effects. The payload (DXd) itself is cell membrane permeable, which means it may enter nearby cells, even those without strong HER2 expression, making it effective in low HER2-expressing cancer cells. This so-called cytotoxic bystander effect may assist in overcoming intratumoral heterogeneity of cancer cells, which is seen in most malignancies, increases with tumor growth and represents a significant barrier to treatment. Even though fewer of the trastuzumab deruxtecan molecules attach to the low HER2-expressing cancer cell, some of the cytotoxic payload can still be delivered to effectively kill the cancer cell. By way of contrast, the payload of trastuzumab emtansine has low membrane permeability and therefore does not exhibit this effect.

Resistance mechanisms to trastuzumab deruxtecan are as of yet, not well defined. Resistance may be acquired by downregulation or loss of HER2 expression, as the antibody-drug conjugate must undergo endocytosis before the payload is released. Defects in internalization, apoptotic dysregulation, and drug efflux pumps can also lead to resistance in other antibody-drug conjugates. ${ }^{16}$ The payload of DS-8201 is not a substrate of p-glycoprotein 
however and therefore is not subject to p-glycoprotein mediated drug efflux. There is some evidence to suggest that resistance to ADCs may develop by reduction in lysosomal proteolytic activity causing the antibody-drug conjugate to accumulate in lysosomes. ${ }^{17}$ Tumor cells may also develop resistance to the topoisomerase I payload.

There are several other antibody-drug conjugates in development. Trastuzumab duocarmazine is composed of trastuzumab linked to a prodrug of duocarmycin, an alkylating agent via a cleavable linker. It has a drug-to-antibody ratio of 2.8:1. The overall response rate in a phase I study in a pretreated breast cancer population was seen to be $33 \%{ }^{18}$ It has shown activity in gastric, ovarian, and endometrial cancers which overexpress HER2 and in the low HER2 population. Phase 1 trials are currently underway of 2 other antibody-drug conjugates targeting HER2, XMT1522 and ARZ788. XMT-1522 is composed of a novel human IgG1 anti-HER2 monoclonal antibody with Auristatin F-hydroxypropylamide as the cytotoxic payload. ARX788 is composed of a HER2-targeted antibody site specifically conjugated to Amberstatin269, a cytotoxic tubulin inhibitor.

\section{Efficacy}

Trastuzumab deruxtecan has shown impressive response rates in early phase studies. The phase I dose-escalation study examined doses of trastuzumab deruxtecan between 0.8 and $8.0 \mathrm{mg} / \mathrm{kg}$ in 24 patients with dose-limiting toxicities assessed over a 21-day cycle. ${ }^{19}$ Based on these results, 2 doses, $5.4 \mathrm{mg} / \mathrm{kg}$ and $6.4 \mathrm{mg} / \mathrm{kg}$ were recommended for the expansion phase. The phase I doseexpansion study evaluated these doses in 118 patients, across 8 hospitals in the United States and Japan. There were 5 patients cohorts: HER2-positive breast cancer after trastuzumab emtansine; HER2-positive gastric or gastroesophageal junction cancer after trastuzumab; HER2-lowexpressing breast cancer (immunohistochemistry $1+$ or $2+$, or insitu hybridization-negative); other HER2-expressing or HER2-mutated solid tumors. In the HER2-positive breast cancer population, the recommended dose was $5.4 \mathrm{mg} / \mathrm{kg}$ and $59.5 \%$ of patients had an objective response (Table 1). ${ }^{14}$

The phase II DESTINY-BREAST01 trial was an openlabel, single-group, multicenter study and included 184 patients with metastatic HER2-positive breast cancer who had undergone prior treatment with trastuzumab emtansine. The median age of the study population was 55 years and $52.7 \%$ had hormone receptor positive disease. This
Table I Patient Demographics and Outcomes in the Phase I and II Trials of Single Agent Trastuzumab Deruxtecan

\begin{tabular}{|l|l|l|}
\hline & $\begin{array}{l}\text { Phase I } \\
\text { Tamura et al } \\
\text { N=II5 }\end{array}$ & $\begin{array}{l}\text { Phase II } \\
\text { DESTINY BREAST } \\
\text { II } \\
\text { N = 184 }\end{array}$ \\
\hline Age, median (range) & $55(47-66)$ & $55(28-96)$ \\
\hline Prior lines of cancer therapy & 7 & 6 \\
\hline Hormone receptor positive & $81(70 \%)$ & $97(53 \%)$ \\
\hline $\begin{array}{l}\text { HER2 expression } \\
\text { IHC 3+ } \\
\text { IHC I+ or 2+, ISH-positive }\end{array}$ & $32(27 \%)$ & $\begin{array}{l}154(83.7 \%) \\
28(15.2 \%)\end{array}$ \\
\hline Objective response rate & $59.5 \%$ & $60.9 \%$ \\
\hline $\begin{array}{l}\text { Median progression free } \\
\text { survival }\end{array}$ & 22.1 months & 16.4 months \\
\hline
\end{tabular}

was a heavily pretreated group as the median number of previous cancer regimens was $6 ; 65.8 \%$ had prior treatment with pertuzumab (Table 1). An objective response rate of $60.9 \%$ was observed. ${ }^{12}$ This is particularly impressive when we consider the response rates to lapatinibcapecitabine (22\%), neratinib-capecitabine $(32.8 \%)$, and tucatinib-capecitabine-trastuzumab $(40.6 \%)$ in similar patient cohorts. In total, $6.0 \%$ achieved a complete response to therapy. The median duration of response was 14.8 months $(95 \%$ CI $13.8,16.9)$, and the median progression-free survival was 16.4 months (95\% CI 12.7, NR). Responses appeared to be consistent across patient subgroups. The median overall survival has not been reached but estimated the overall survival was $93.9 \%$ and $86.2 \%$ at 6 and 12 months, respectively. This level of activity appears higher than has been seen with other antibody-drug conjugates, e.g. trastuzumab emtansine in a similar patient population (Table 2). Importantly, trastuzumab deruxtecan was also shown to be effective in those with pretreated or asymptomatic brain metastases, with a median progression-free survival in that cohort of 18.1 months $(95 \%$ CI $6.7,18.1)$. The demographics and outcomes of patients in the phase II trials and I are outlined in Table 1.

Interestingly, the phase I trial included 54 patients with low HER2-expressing breast cancer. This was reported separately by Modi et al and showed an overall response rate of $37 \%{ }^{20}$ These patients were mostly hormone receptor positive $(87 \%)$ and $83 \%$ had 5 more prior lines of treatment. Notably, only $30 \%$ had prior treatment with 
Table 2 Comparison of Efficacy and Toxicity of Single Agent Trastuzumab Deruxtecan (Phase II) and Trastuzumab Emtansine (Phase III)

\begin{tabular}{|c|c|c|}
\hline & $\begin{array}{l}\text { Trastuzumab } \\
\text { Deruxtecan }^{12} \\
N=184 \\
N \text { (\%) }\end{array}$ & $\begin{array}{l}\text { Trastuzumab } \\
\text { Emtansine }^{6} \\
N=495 \\
N(\%)\end{array}$ \\
\hline Age, median (range) & $55(28-96)$ & $53(25-84)$ \\
\hline Age $\geq 65$ & $64(25.3 \%)$ & $138(27.9 \%)$ \\
\hline \multicolumn{3}{|l|}{ Performance status } \\
\hline $\begin{array}{l}0 \\
1 \\
2\end{array}$ & $\begin{array}{l}102(55.4 \%) \\
81(44 \%) \\
I(0.5 \%)\end{array}$ & $\begin{array}{l}299(60 \%) \\
194(39 \%) \\
0(0 \%)\end{array}$ \\
\hline $\begin{array}{l}\geq 2 \text { prior treatments for } \\
\text { metastatic disease }\end{array}$ & $184(100 \%)$ & 191 (39\%) \\
\hline $\begin{array}{l}\text { Hormone receptor } \\
\text { positive }\end{array}$ & 97 (52.7\%) & $282(57 \%)$ \\
\hline Objective response rate & $60.9 \%$ & $43.6 \%$ \\
\hline Progression free survival & 16.4 months & 9.6 months \\
\hline $\begin{array}{l}\text { Median duration of } \\
\text { response }\end{array}$ & 14.8 months & 12.6 months \\
\hline \multicolumn{3}{|l|}{$\begin{array}{l}\text { Important toxicities (all } \\
\text { grades) }\end{array}$} \\
\hline LVEF reduced & $3(1.6 \%)$ & $8(1.7 \%)$ \\
\hline Pneumonitis & $25(13.6 \%)$ & Not Reported \\
\hline Increased AST & $26(14.1 \%)$ & $46(9.4 \%)$ \\
\hline Thrombocytopenia & 39 (2I.2\%) & 137 (28\%) \\
\hline
\end{tabular}

a CDK4/6 inhibitor. The median duration of response was 10.4 months and the median overall survival was 29.4 months. This effect is notable, as other HER2-targetted therapies including trastuzumab and trastuzumab emtansine have failed to show any benefit in HER2-low disease. $^{21,22}$ The effect in this cohort may be due to the high drug-to-antibody ratio and the cytotoxic bystander effect as noted above.

Trastuzumab deruxtecan has also shown efficacy in other cancer types that overexpress HER2 or have HER2 mutations. In a randomized phase II study in pretreated metastatic gastric cancer patients, there was an objective response rate of $51 \%$ with Trastuzumab deruxtecan vs $12 \%$ for chemotherapy and an overall survival of 12.5 months vs 8.4 months compared with chemotherapy (HR 0.59). ${ }^{23}$ Other HER2-directed therapies such as pertuzumab and lapatinib have been broadly ineffective in this setting. A separate cohort in the phase I dose-expansion study looked at HER2 overexpressing non-breast/nongastric solid tumors and HER2 mutant solid tumors. ${ }^{24}$ This included 2 patients with HER2-mutated nonamplified breast cancer. In the subgroup comprising HER2-mutated breast cancer and HER2-amplified salivary gland, biliary tract, and endometrial cancer there was an overall response rate of $27.3 \% .{ }^{24}$ The response rate in the 20 patients with colorectal cancer was disappointing at just $5 \%$. The subset of patients with HER2-mutated non-small cell lung cancer exhibited more impressive response rates and therefore this was further studied in the phase II DESTINYLUNG01 trial. Here, patients with previously treated, metastatic non-squamous non-small cell lung cancer were treated with $6.4 \mathrm{mg} / \mathrm{kg}$ trastuzumab deruxtecan and had an impressive overall response rate of $61.9 \%$, similar to that seen in breast cancer population, with median treatment duration of 7.7 months.

\section{Safety}

Given the potency and high drug-to-antibody ratio of trastuzumab deruxtecan, its safety profile is paramount to consider its broad clinical use. As outlined, the payload of trastuzumab deruxtecan is a topoisomerase I inhibitor which is delivered selectively to HER2-expressing tissues. However, HER2 is not just expressed on tumor cells, but also on cell membranes of epithelial cells, heart, and skeletal muscle cells so these cells may be adversely affected.

In the phase I trial, all 115 that received at least one dose of trastuzumab deruxtecan had at least one adverse event (AE) during treatment. Of these, 50\% had a grade 3 or higher AE. ${ }^{14}$ Treatment-emergent adverse events led to discontinuation of the drug in $11 \%$ of patients. Similar rates were seen in the phase II trial, with $57 \%$ experiencing a grade 3 or higher $\mathrm{AE}$ and a discontinuation rate of $15.2 \% .^{12}$ The most common adverse event was nausea, which occurred in $>70 \%$ of patients, but was mostly low grade. Similarly, vomiting, diarrhea, constipation, anorexia, and fatigue occurred in between 30 and $50 \%$ of patients at relatively low grades with very few $\geq$ grade 3 events. Hematological toxicities were frequent, with grade 3 or higher neutropenia, anemia, and thrombocytopenia seen in $20.7 \%, 8.7 \%$, and $4.3 \%$ of patients, respectively, in the DESTINY-BREAST01 trial. Rates of neutropenia were higher here than the rate of $11 \%$ in the phase I trial. Other antibody-drug conjugates have 


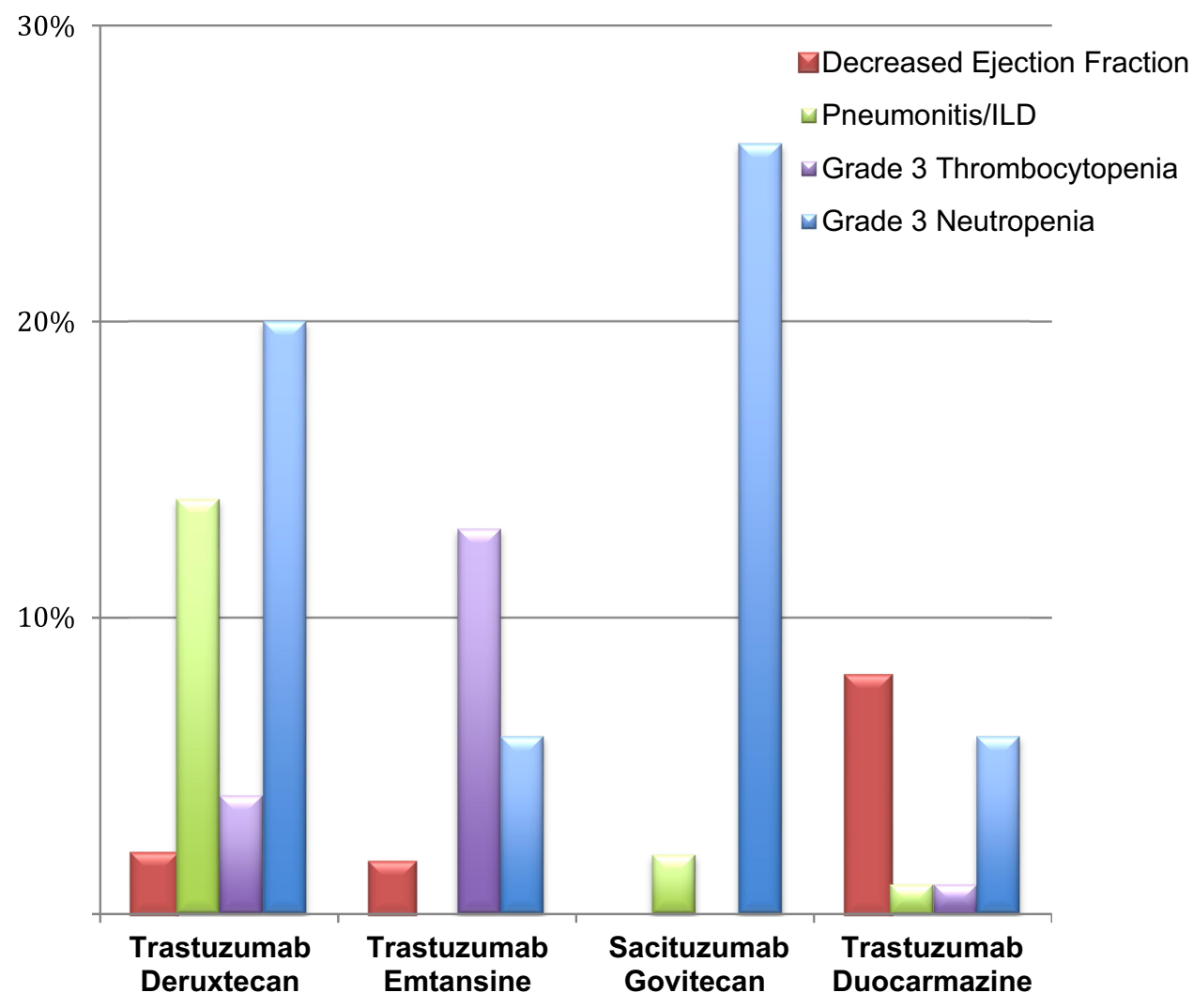

Figure I Relative frequency of side effects of antibody-drug conjugates.

also shown high rates of hematological toxicity, for example, trastuzumab emtansine is associated with significant thrombocytopenia (Figure 1). ${ }^{6,25}$ Sacituzumab govitecan, another antibody-drug conjugate targeting Trop-2 in triple-negative breast cancer showed high rates of neutropenia in a phase II study with $26 \%$ of patients experiencing grade $\geq 3$ neutropenia (Figure 1). ${ }^{26}$

The most significant and potentially fatal adverse event that was observed with trastuzumab deruxtecan was that of interstitial lung disease (ILD). This was reported in $13.6 \%$ of patients in the phase II trial, including $4(2.2 \%)$ patients who died due to lung injury. ${ }^{12}$ The majority of cases were grade 1 and 2, with one grade 3 and no grade 4 events. For the fatal cases, time of onset was 63-148 days and time from diagnosis of ILD to death was 9-60 days. The rates of ILD with trastuzumab deruxtecan in other cancer types are similar. In the phase II gastric cancer trial, the rate of ILD was found to be $10 \%$ (12 patients). Most were grade 1-2, with two grade 3 events, one grade 4 event, and no deaths. Similarly, in the DESTINY-LUNG01 study, $11.9 \%$ of patients had developed interstitial lung disease at interim analysis. All of these cases were grade 1-2. In a pooled analysis of 665 subjects who had received at least one dose of trastuzumab deruxtecan across 7 studies, investigators reported an ILD rate of $9.9 \% .{ }^{27}$ They found that higher doses of trastuzumab deruxtecan and Japanese origin were associated with an increased risk of ILD.

Given the potential severity, ILD or pneumonitis should be suspected if a patient develops acute onset of new or worsening dyspnea, cough, or fever. Current recommendations are to delay treatment, perform initial evaluations eg, high-resolution CT, oxygen saturations, respiratory review, and pulmonary function tests as appropriate. Consideration should be given to early initiation of steroids. ${ }^{24}$ If the ILD is confirmed to be grade 1, trastuzumab deruxtecan should be restarted only if the event is fully resolved, and may require dose reduction. For grade 2 or greater, treatment should be permanently discontinued. ${ }^{28}$

The mechanism of lung injury is not well defined at present. It may be due to the payload as other topoisomerase inhibitors (eg, topotecan) have been associated with interstitial lung disease. ${ }^{29}$ Other antibody- 
drug conjugates have also been associated with pulmonary toxicity. Trastuzumab emtansine is now known to be associated with interstitial lung disease, though this was not initially identified as a treatmentrelated adverse event in the TH3RESA and EMILIA studies (Figure 1). ${ }^{6,25}$ Pneumonitis was also seen with trastuzumab duocarmazine however in smaller numbers, being reported in $8 \%$ of the dose-escalation cohort and $1 \%$ of the dose-expansion cohort.

In contrast to some other HER2-targeted agents, particularly trastuzumab, there appears to be a low rate of left ventricular dysfunction with trastuzumab deruxtecan, reported in just $1.6 \%$ of patients to date. All were asymptomatic and recovered after interruption of study treatment. By comparison, the HER2 antibody-drug conjugate, trastuzumab duocarmazine reported $8 \%$ rate of left ventricular dysfunction in a phase I study. ${ }^{18}$ Trastuzumab emtansine had similar rates of left ventricular dysfunction to trastuzumab deruxtecan (Table 2). The most significant side effects of trastuzumab deruxtecan compared to other antibody-drug conjugates are depicted in Figure 1.

\section{Discussion}

Trastuzumab deruxtecan is a promising new agent in heavily pretreated HER2-positive metastatic breast cancer. Response rates are impressive and preliminary survival data are promising. Its activity in a broad range of HER2 expressing and mutated solid tumors has led to a plethora of new clinical trials and potential for widespread use. However, we must remember that the available data is still phase II, and we must await phase III randomized data to determine the true value and treatment line of trastuzumab deruxtecan. The responding population could represent a subset of HER2-addicted patients who respond for a time to all agents, and may not be representative of a truly resistant population who are in need of a new agent.

The safety signal of interstitial lung disease may prove to be a limiting factor in phase III studies. Patients will require careful monitoring and swift intervention should symptoms appear. The lack of effect on cardiac function is positive, but the exact incidence of cardiotoxicity may become more apparent in larger trials. Tolerability may become the biggest determining factor when comparing to the other new treatments.
Given the number of new HER2-targeted agents coming on stream, determining the optimum sequencing for these new medications will be a challenge for physicians. Based on current evidence, trastuzumab deruxtecan could be considered in the third line. With the recent phase III data on tucatinib, particularly its intracranial efficacy and tolerability; it may be the preferred agent in the third line until there is further phase 3 efficacy and safety data for trastuzumab deruxtecan. In heavily pretreated fit patients who have had previous tyrosine kinase inhibitors, it is an attractive option. The paradigm is likely to change for some patients in the coming years however, as trastuzumab emtansine moves into the adjuvant setting on the back of the KATHERINE study which showed $50 \%$ reduction in risk of recurrence in patients who had residual disease following neoadjuvant therapy. ${ }^{30}$ This may result in trastuzumab deruxtecan being used in earlier lines of therapy. Trastuzumab deruxtecan is itself being investigated in the phase III DESTINY-BREAST05 trial comparing it to trastuzumab emtansine in patients with an incomplete response to neoadjuvant therapy.

There are numerous other ongoing and planned trials investigating trastuzumab deruxtecan as outlined in Table 3. Multiple trials are examining combination treatment with immunotherapy in both HER2-positive and HER2-low disease. Preclinical data in mouse models have shown combination therapy with anti-PD1 agents to be more effective than either monotherapy. ${ }^{31}$ Initial data of trastuzumab deruxtecan in combination with nivolumab were presented at San Antonio Breast Cancer Symposium 2020 and showed a preliminary response rate of 59\% in HER2-positive and $38 \%$ in HER2-low patients. The combination saw a higher treatment discontinuation rate of $18.8 \%$ due to adverse events than with either monotherapy; $43.8 \%$ of patients developed grade 3 or higher adverse events, and $10.4 \%$ developed treatment-related interstitial lung disease. It is also being investigated in combination therapy with endocrine therapy for hormone receptor positive disease in both metastatic and neoadjuvant settings. The phase II HER2CLIMB04 will examine combination treatment with tucatinib in pretreated metastatic breast cancer (Table 3).

In conclusion, trastuzumab deruxtecan is an exciting antibody-drug conjugate with impressive activity in heavily pretreated HER2-positive breast cancer. The safety profile appears manageable, although caution is needed regarding the risk of interstitial lung disease. 
Table 3 Ongoing Phase I-III Clinical Trials in Breast Cancer with Trastuzumab Deruxtecan

\begin{tabular}{|c|c|c|c|c|c|c|}
\hline Trial & Status & Setting & Line & Population & Comparator & Combined with \\
\hline \multicolumn{7}{|l|}{ PHASE I } \\
\hline NCT03523572 & Recruiting & Metastatic & $\begin{array}{l}\text { Second } \\
\text { or later }\end{array}$ & $\begin{array}{l}\text { HER2+ previously treated with } 2 \text { or more } \\
\text { anti-HER2 based regimens }\end{array}$ & $\mathrm{N} / \mathrm{A}$ & Nivolumab \\
\hline NCT0404270I & Recruiting & Metastatic & $\begin{array}{l}\text { Third } \\
\text { or later }\end{array}$ & $\begin{array}{l}\text { HER2 + previously treated with T-DMI and } \\
\text { low HER2 who failed standard treatments }\end{array}$ & $\mathrm{N} / \mathrm{A}$ & Pembrolizumab \\
\hline $\begin{array}{l}\text { DESTINY- } \\
\text { BREAST } 08\end{array}$ & $\begin{array}{l}\text { Not yet } \\
\text { recruiting }\end{array}$ & Metastatic & $\begin{array}{l}\text { Second } \\
\text { or later }\end{array}$ & $\begin{array}{l}\text { Low HER2 expressing breast cancer with at } \\
\text { least one prior line of chemotherapy }\end{array}$ & $\mathrm{N} / \mathrm{A}$ & $\begin{array}{l}\text { Chemotherapy, } \\
\text { immunotherapy or } \\
\text { endocrine tx }\end{array}$ \\
\hline \multicolumn{7}{|l|}{ PHASE I/II } \\
\hline BEGONIA & Recruiting & Metastatic & $\begin{array}{l}\text { First } \\
\text { line }\end{array}$ & $\begin{array}{l}\text { Triple negative breast cancer with low HER2 } \\
\text { expression }\end{array}$ & $\mathrm{N} / \mathrm{A}$ & Durvalumab \\
\hline $\begin{array}{l}\text { DESTINY- } \\
\text { BREAST } 07\end{array}$ & $\begin{array}{l}\text { Not yet } \\
\text { recruiting }\end{array}$ & Metastatic & $\begin{array}{l}\text { Second } \\
\text { or later }\end{array}$ & $\begin{array}{l}\text { HER2+ breast cancer both previously } \\
\text { treated and with no prior treatment }\end{array}$ & $\mathrm{N} / \mathrm{A}$ & $\begin{array}{l}\text { Durvalumab or } \\
\text { paclitaxel or } \\
\text { pertuzumab }\end{array}$ \\
\hline \multicolumn{7}{|l|}{ PHASE II } \\
\hline $\begin{array}{l}\text { DESTINY- } \\
\text { BREAST OI }\end{array}$ & Completed & Metastatic & $\begin{array}{l}\text { Third } \\
\text { or later }\end{array}$ & $\begin{array}{l}\text { HER2+ breast cancer previously treated } \\
\text { with T-DMI }\end{array}$ & $\mathrm{N} / \mathrm{A}$ & $N / A$ \\
\hline DEBBRAH & Recruiting & Metastatic & $\begin{array}{l}\text { Second } \\
\text { or later }\end{array}$ & $\begin{array}{l}\text { HER2 + breast cancer with untreated/ } \\
\text { treated brain metastases or leptomeningeal } \\
\text { disease }\end{array}$ & $\mathrm{N} / \mathrm{A}$ & N/A \\
\hline HER2CLIMB04 & Recruiting & Metastatic & $\begin{array}{l}\text { Third } \\
\text { or later }\end{array}$ & $\begin{array}{l}\text { HER } 2+\text { breast cancer previously treated } \\
\text { with } 2 \text { or more anti-HER } 2 \text { based regimens }\end{array}$ & $\mathrm{N} / \mathrm{A}$ & Tucatinib \\
\hline NCT04553770 & $\begin{array}{l}\text { Not yet } \\
\text { recruiting }\end{array}$ & $\begin{array}{l}\text { Neo- } \\
\text { adjuvant }\end{array}$ & & $\begin{array}{l}\text { HER2 low, HR+ early breast cancer which is } \\
\text { deemed operable }\end{array}$ & $\mathrm{N} / \mathrm{A}$ & Anastrazole \\
\hline \multicolumn{7}{|l|}{ PHASE III } \\
\hline $\begin{array}{l}\text { DESTINY- } \\
\text { BREAST } 03\end{array}$ & $\begin{array}{l}\text { Completed } \\
\text { accrual }\end{array}$ & Metastatic & $\begin{array}{l}\text { Second } \\
\text { or later }\end{array}$ & $\begin{array}{l}\text { HER2 + breast cancer previously treated } \\
\text { with trastuzumab and taxane }\end{array}$ & T-DMI & $N / A$ \\
\hline $\begin{array}{l}\text { DESTINY- } \\
\text { BREAST } 04\end{array}$ & Recruiting & Metastatic & $\begin{array}{l}\text { Second } \\
\text { or later }\end{array}$ & $\begin{array}{l}\text { Low HER2 expressing breast cancer } \\
\text { previously treated with chemotherapy }\end{array}$ & $\begin{array}{l}\text { Physician's } \\
\text { Choice }\end{array}$ & N/A \\
\hline $\begin{array}{l}\text { DESTINY- } \\
\text { BREAST } 06\end{array}$ & Recruiting & Metastatic & $\begin{array}{l}\text { Second } \\
\text { or later }\end{array}$ & $\begin{array}{l}\text { Low HER2, HR+ breast cancer following } \\
\text { progression on endocrine therapy }\end{array}$ & $\begin{array}{l}\text { Physician's } \\
\text { Choice }\end{array}$ & $\mathrm{N} / \mathrm{A}$ \\
\hline $\begin{array}{l}\text { DESTINY- } \\
\text { BREAST } 02\end{array}$ & Recruiting & Metastatic & $\begin{array}{l}\text { Third } \\
\text { or later }\end{array}$ & $\begin{array}{l}\text { HER2+ breast cancer previously treated } \\
\text { with T-DMI }\end{array}$ & T-DMI & $N / A$ \\
\hline $\begin{array}{l}\text { DESTINY- } \\
\text { BREAST } 05\end{array}$ & $\begin{array}{l}\text { Not yet } \\
\text { recruiting }\end{array}$ & Adjuvant & & $\begin{array}{l}\text { HER2+ early breast cancer with residual } \\
\text { disease post neoadjuvant treatment }\end{array}$ & T-DMI & $\mathrm{N} / \mathrm{A}$ \\
\hline
\end{tabular}

Abbreviations: HER2, human epidermal receptor; HR, hormone receptor; T-DMI, trastuzumab emtansine.

Ongoing clinical trials are investigating this exciting agent in earlier lines of therapy, including in patients with curable breast cancer in an attempt to individualize treatment for patients.

\section{Disclosure}

PG Morris has received travel support and honoraria from Roche; travel support from Novartis and Amgen; reports personal fees from Astellas and Pfizer, grants from Teva, 
BMS, Astra Zeneca, and Genomic Health, during the conduct of the study. The authors report no other conflicts of interest in this work.

\section{References}

1. Slamon DJ, Leyland-Jones B, Shak S, et al. Use of chemotherapy plus a monoclonal antibody against her2 for metastatic breast cancer that overexpresses HER2. $N$ Engl J Med. 2001;344:783-792. doi:10.1056/NEJM200103153441101

2. Deluche E, Antoine A, Bachelot T, et al. Contemporary outcomes of metastatic breast cancer among 22,000 women from the multicentre ESME cohort 2008-2016. Eur J Cancer. 2020;129:60-70. doi:10.1016/j.ejca.2020.01.016

3. Murphy CG, Morris PG. Recent advances in novel targeted therapies for HER2-positive breast cancer. Anticancer Drugs. 2012;23:765-776. doi:10.1097/CAD.0b013e328352d292

4. Gleeson JP, Keegan NM, Morris PG. Adding pertuzumab to trastuzumab and taxanes in HER2 positive breast cancer. Expert Opin Biol Ther. 2018;18:251-262. doi:10.1080/14712598.2018.1410132

5. Swain SM, Baselga J, Kim SB, et al. Pertuzumab, trastuzumab, and docetaxel in HER2-positive metastatic breast cancer. $N$ Engl J Med. 2014. doi:10.1056/NEJMoa1413513

6. Verma S, Miles D, Gianni L, et al. Trastuzumab emtansine for HER2-positive advanced breast cancer. $N$ Engl $J$ Med. 2012;367:1783-1791. doi:10.1056/NEJMoa1209124

7. Geyer CE, Forster J, Lindquist D, et al. Lapatinib plus capecitabine for HER2-positive advanced breast cancer. $N$ Engl $J$ Med. 2006;355:2733-2743. doi:10.1056/NEJMoa064320

8. Cameron D, Casey M, Oliva C, Newstat B, Imwalle B, Geyer CE. Lapatinib plus capecitabine in women with HER-2-positive advanced breast cancer: final survival analysis of a phase III randomized trial. Oncologist. 2010;15:924-934. doi:10.1634/theoncologist.2009-0181

9. Saura C, Oliveira M, Feng Y-H, et al. Neratinib + capecitabine versus lapatinib + capecitabine in patients with HER2+ metastatic breast cancer previously treated with $\geq 2$ HER2-directed regimens: findings from the multinational, randomized, phase III NALA trial. $J$ Clin Oncol. 2019;37:1002. doi:10.1200/jco.2019.37.15_suppl.1002

10. Murthy RK, Loi S, Okines A, et al. Tucatinib, trastuzumab, and capecitabine for HER2-positive metastatic breast cancer. $N$ Engl J Med. 2020;597-609. doi:10.1056/NEJMoa1914609.

11. Rugo HS, Im S-A, Wright GLS, et al. SOPHIA primary analysis: a phase 3 (P3) study of margetuximab (M) + chemotherapy (C) versus trastuzumab $(\mathrm{T})+\mathrm{C}$ in patients (pts) with HER2 + metastatic (met) breast cancer (MBC) after prior anti-HER2 therapies (Tx). J Clin Oncol. 2019;37:1000. doi:10.1200/jco.2019.37.15_suppl.1000

12. Modi S, Saura C, Yamashita T, et al. Trastuzumab deruxtecan in previously treated HER2-positive breast cancer. $N$ Engl J Med. 2020;382:610-621. doi:10.1056/NEJMoa1914510

13. Xu Z, Guo D, Jiang Z, et al. Novel HER2-targeting antibody-drug conjugates of trastuzumab beyond T-DM1 in breast cancer: trastuzumab deruxtecan(DS-8201a) and (Vic-)trastuzumab duocarmazine (SYD985). Eur J Med Chem. 2019;183:111682. doi:10.1016/j. ejmech.2019.111682

14. Tamura K, Tsurutani J, Takahashi S, et al. Trastuzumab deruxtecan (DS-8201a)in patients with advanced HER2-positive breast cancer previously treated with trastuzumab emtansine: a dose-expansion, phase 1 study. Lancet Oncol. 2019;20:816-826. doi:10.1016/S14702045(19)30097-X

15. Andrikopoulou A, Zografos E, Liontos M, Koutsoukos K, Dimopoulos MA, Zagouri F. Trastuzumab deruxtecan (DS-8201a): the latest research and advances in breast cancer. Clin Breast Cancer. 2020. doi:10.1016/j.clbc.2020.08.006
16. García-Alonso S, Ocaña A, Pandiella A. Resistance to antibody-drug conjugates. Cancer Res. 2018;78:2159-2165. doi:10.1158/00085472.CAN-17-3671

17. Ríos-Luci C, García-Alonso S, Díaz-Rodríguez E, et al. Resistance to the antibody-drug conjugate T-DM1 is based in a reduction in lysosomal proteolytic activity. Cancer Res. 2017;77:4639-4651. doi:10.1158/0008-5472.CAN-16-3127

18. Banerji U, van Herpen CML, Saura C, et al. Trastuzumab duocarmazine in locally advanced and metastatic solid tumours and HER2-expressing breast cancer: a phase 1 dose-escalation and dose-expansion study. Lancet Oncol. 2019;20:1124-1135. doi:10.1016/S1470-2045(19)30328-6

19. Doi T, Shitara K, Naito Y, et al. Safety, pharmacokinetics, and antitumour activity of trastuzumab deruxtecan (DS-8201), a HER2-targeting antibody-drug conjugate, in patients with advanced breast and gastric or gastro-oesophageal tumours: a phase 1 dose-escalation study. Lancet Oncol. 2017;18:1512-1522. doi:10.1016/S1470-2045(17)30604-6

20. Modi S, Park H, Murthy RK, et al. Antitumor activity and safety of trastuzumab deruxtecan in patients with HER2-low-expressing advanced breast cancer: results from a phase $\mathrm{Ib}$ study. $J$ Clin Oncol. 2020;38:1887-1896. doi:10.1200/JCO.19.02318

21. Yazaki S, Hashimoto J, Ogita S, Nakano E, Yamauchi T. Lower response to T-DM1 in metastatic breast cancer patients with HER2 IHC score of 2 and FISH positive compared with IHC score of 3. Ann Oncol. 2017;28:v102-v103. doi:10.1093/annonc/mdx365.071

22. Fehrenbacher L, Cecchini RS, Geyer CE, et al. NSABP B-47/NRG oncology phase III randomized trial comparing adjuvant chemotherapy with or without trastuzumab in high-risk invasive breast cancer negative for HER2 by FISH and with IHC 1+ or 2+. J Clin Oncol. 2020;38:444-453. doi:10.1200/JCO.19.01455

23. Shitara K, Bang Y-J, Iwasa S, et al. Trastuzumab deruxtecan in previously treated HER2-positive gastric cancer. $N$ Engl $J$ Med. 2020;382:2419-2430. doi:10.1056/nejmoa2004413

24. Tsurutani J, Iwata H, Krop I, et al. Targeting her2 with trastuzumab deruxtecan: a dose-expansion, phase i study in multiple advanced solid tumors. Cancer Discov. 2020;10:688-701. doi:10.1158/21598290.CD-19-1014

25. Krop IE, Kim SB, Martin AG, et al. Trastuzumab emtansine versus treatment of physician's choice in patients with previously treated HER2-positive metastatic breast cancer (TH3RESA): final overall survival results from a randomised open-label phase 3 trial. Lancet Oncol. 2017;18:743-754. doi:10.1016/S1470-2045(17)30313-3

26. Bardia A, Mayer IA, Vahdat LT, et al. Sacituzumab govitecan-hziy in refractory metastatic triple-negative breast cancer. $N$ Engl $J$ Med. 2019;380:741-751. doi:10.1056/NEJMoa1814213

27. Powell C, Camidge D, Gemma A, et al. Abstract P6-17-06: characterization, monitoring and management of interstitial lung disease in patients with metastatic breast cancer: analysis of data available from multiple studies of DS-8201a, a HER2-targeted antibody drug conjugate with a topoisomera. Cancer Res. 2019. doi:10.1158/15387445.sabcs18-p6-17-06

28. Kubo K, Azuma A, Kanazawa M, et al. Consensus statement for the diagnosis and treatment of drug-induced lung injuries. Respir Investig. 2013;51:260-277. doi:10.1016/j.resinv.2013.09.001

29. Enomoto Y, Inui N, Imokawa S, et al. Safety of topotecan monotherapy for relapsed small cell lung cancer patients with pre-existing interstitial lung disease. Cancer Chemother Pharmacol. 2015;76:499-505. doi:10.1007/s00280-015-2816-6

30. Von Minckwitz G, Huang CS, Mano MS, et al. Trastuzumab emtansine for residual invasive HER2-positive breast cancer. $N$ Engl J Med. 2019;380:617-628. doi:10.1056/NEJMoa1814017

31. Iwata TN, Ishii C, Ishida S, Ogitani Y, Wada T, Agatsuma T. A HER2-targeting antibody-drug conjugate, trastuzumab deruxtecan (DS-8201a), enhances antitumor immunity in a mouse model. Mol Cancer Ther. 2018;17:1494-1503. doi:10.1158/1535-7163.MCT-170749 


\section{Publish your work in this journal}

Breast Cancer - Targets and Therapy is an international, peer-reviewed open access journal focusing on breast cancer research, identification of therapeutic targets and the optimal use of preventative and integrated treatment interventions to achieve improved outcomes, enhanced survival and quality of life for the cancer patient.
The manuscript management system is completely online and includes a very quick and fair peer-review system, which is all easy to use. Visit http://www.dovepress.com/testimonials.php to read real quotes from published authors. 